\title{
Fatty Liver Index and Lipid Accumulation Product Can Predict Metabolic Syndrome in Subjects without Fatty Liver Disease
}

\author{
Yuan-Lung Cheng, ${ }^{1,2}$ Yuan-Jen Wang, ${ }^{2,3}$ Keng-Hsin Lan, ${ }^{2,4,5}$ Teh-Ia Huo, \\ Yi-Hsiang Huang, ${ }^{4,6}$ Chien-Wei Su, ${ }^{2,4}$ Wei-Yao Hsieh, ${ }^{4}$ Ming-Chih Hou, ${ }^{2,4}$ Han-Chieh Lin, ${ }^{2,4}$ \\ Fa-Yauh Lee, ${ }^{2,4}$ Jaw-Ching $\mathrm{Wu}^{6,7}$ and Shou-Dong Lee ${ }^{2,8}$ \\ ${ }^{1}$ Taipei Municipal Gan-Dau Hospital, Taipei, Taiwan \\ ${ }^{2}$ Faculty of Medicine, School of Medicine, National Yang-Ming University, Taipei, Taiwan \\ ${ }^{3}$ Healthcare Center, Taipei Veterans General Hospital, Taipei, Taiwan \\ ${ }^{4}$ Division of Gastroenterology and Hepatology, Department of Medicine, Taipei Veterans General Hospital, Taipei, Taiwan \\ ${ }^{5}$ Department and Institute of Pharmacology, National Yang-Ming University, Taipei, Taiwan \\ ${ }^{6}$ Institute of Clinical Medicine, School of Medicine, National Yang-Ming University, Taipei, Taiwan \\ ${ }^{7}$ Division of Translational Research, Department of Medical Research, Taipei Veterans General Hospital, Taipei, Taiwan \\ ${ }^{8}$ Division of Gastroenterology, Department of Medicine, Cheng Hsin General Hospital, Taipei, Taiwan \\ Correspondence should be addressed to Chien-Wei Su; cwsu2@vghtpe.gov.tw
}

Received 9 July 2016; Revised 9 November 2016; Accepted 20 November 2016; Published 17 January 2017

Academic Editor: Kazuhiko Uchiyama

Copyright (c) 2017 Yuan-Lung Cheng et al. This is an open access article distributed under the Creative Commons Attribution License, which permits unrestricted use, distribution, and reproduction in any medium, provided the original work is properly cited.

\begin{abstract}
Background. Fatty liver index (FLI) and lipid accumulation product (LAP) are indexes originally designed to assess the risk of fatty liver and cardiovascular disease, respectively. Both indexes have been proven to be reliable markers of subsequent metabolic syndrome; however, their ability to predict metabolic syndrome in subjects without fatty liver disease has not been clarified. Methods. We enrolled consecutive subjects who received health check-up services at Taipei Veterans General Hospital from 2002 to 2009. Fatty liver disease was diagnosed by abdominal ultrasonography. The ability of the FLI and LAP to predict metabolic syndrome was assessed by analyzing the area under the receiver operating characteristic (AUROC) curve. Results. Male sex was strongly associated with metabolic syndrome, and the LAP and FLI were better than other variables to predict metabolic syndrome among the 29,797 subjects. Both indexes were also better than other variables to detect metabolic syndrome in subjects without fatty liver disease (AUROC: 0.871 and 0.879, resp.), and the predictive power was greater among women. Conclusion. Metabolic syndrome increases the cardiovascular disease risk. The FLI and LAP could be used to recognize the syndrome in both subjects with and without fatty liver disease who require lifestyle modifications and counseling.
\end{abstract}

\section{Introduction}

Metabolic syndrome comprises risk factors of cardiovascular disease and type 2 diabetes mellitus (DM), including central obesity, dyslipidemia, and high blood pressure and fasting glucose [1]. Nonalcoholic fatty liver disease (NAFLD) used to be considered an incidental pathologic finding in type 2 $\mathrm{DM}$ and obesity but was found to be strongly associated with features of subsequent metabolic syndrome and was even included in the definition of metabolic syndrome $[2,3]$.
Using data from the general population of northern Italy, the fatty liver index (FLI), an algorithm based on triglyceride (TG) concentration, gamma-glutamyl transferase (GGT) level, body mass index (BMI), and waist circumference (WC), was developed to predict the risk of fatty liver disease in the general population [4]. The FLI has been validated by several studies and has been proven to have a strong association with hypertension and type 2 DM [5-9]. As cardiovascular disease, NAFLD, and metabolic syndrome are closely related, the FLI 


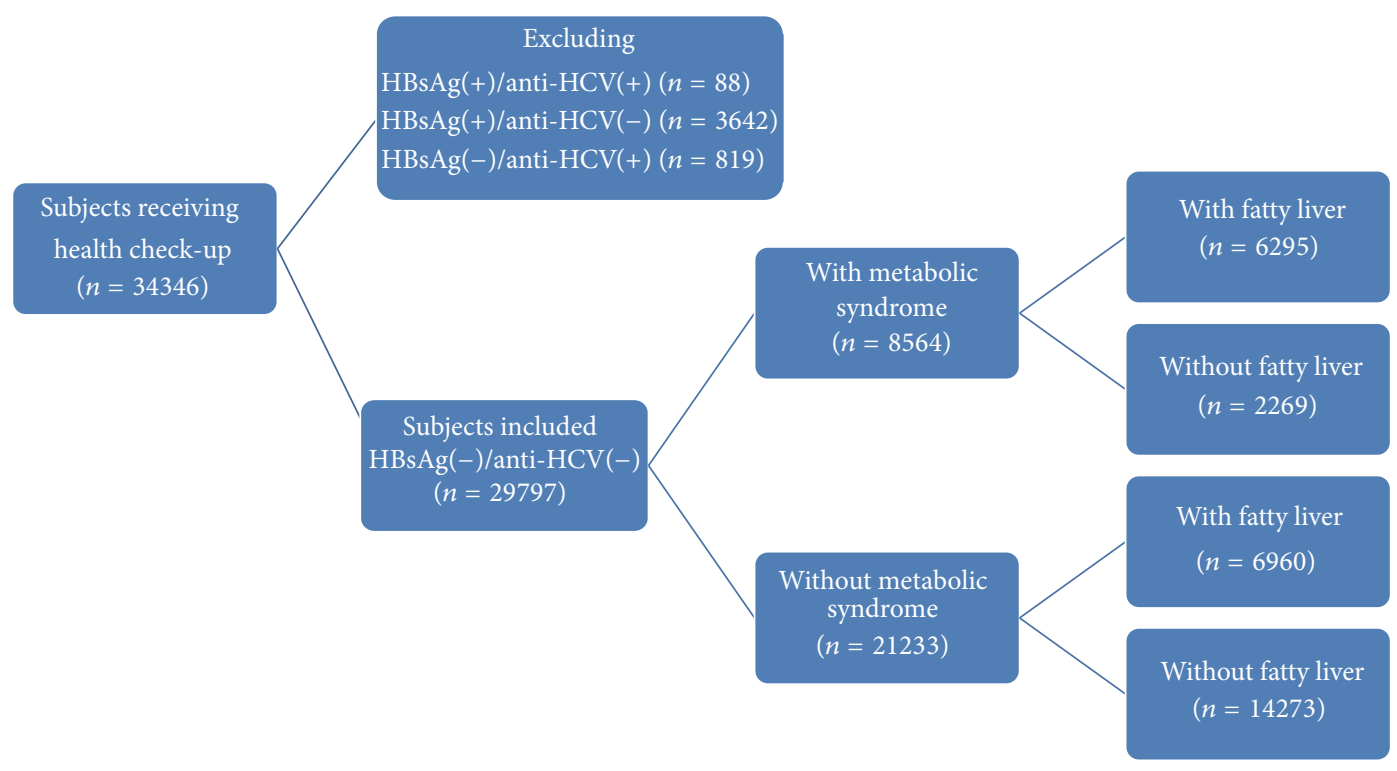

FIGURE 1: The algorithm for patient selection.

was also found to have a strong association with metabolic syndrome $[10,11]$.

The lipid accumulation product (LAP) is an index based on two components, WC and TG concentration, and was designed to indicate the risk of cardiovascular disease. As the LAP shares two of the five components of metabolic syndrome, it has been found to be a reliable tool to detect metabolic syndrome as well [12-14].

However, the ability of the FLI and LAP to predict metabolic syndrome in subjects without fatty liver disease, a group of people with cardiovascular risk as well, has not been clarified. Therefore, the present study aimed to determine the association between the two indexes and metabolic syndrome and to further explore their ability to predict metabolic syndrome in subjects without fatty liver disease in a largescale cohort in Taiwan.

\section{Materials and Methods}

2.1. Study Population. In total, 34,346 subjects received health check-up services provided by internists in the Healthcare Center without hospitalization at the Taipei Veterans General Hospital from 2002 to 2009 [15-18]. Those with hepatitis B virus (HBV) infection, hepatitis $\mathrm{C}$ virus (HCV) infection, or $\mathrm{HBV} / \mathrm{HCV}$ dual infections were excluded, and the remaining subjects were analyzed (Figure 1). All the subjects underwent complete clinical evaluations, laboratory examinations, and abdominal ultrasonography. The BMI was calculated as body weight (in kilograms) divided by the square of body height (in meters). Blood pressure (BP) was measured after the subjects had been seated for more than 5 minutes. The means of three consecutive readings were recorded as the systolic and diastolic BP with a difference in systolic $\mathrm{BP}<10 \mathrm{mmHg}$. A diagnosis of metabolic syndrome was made when three of the following five abnormal findings were met according to the joint interim statement of the International Diabetes Federation Task Force on Epidemiology and Prevention [1]: elevated waist circumference (WC, men $\geq 90 \mathrm{~cm}$ or women $\geq$ $80 \mathrm{~cm}$ ); TG $\geq 150 \mathrm{mg} / \mathrm{dL}$; low high-density lipoprotein cholesterol (men $<40 \mathrm{mg} / \mathrm{dL}$ or women $<50 \mathrm{mg} / \mathrm{dL}$ ); systolic BP $\geq 130 \mathrm{mmHg}$ and/or diastolic $\mathrm{BP} \geq 85 \mathrm{mmHg}$; and fasting glucose $\geq 100 \mathrm{mg} / \mathrm{dL}$. Impaired fasting glucose (IFG) was defined as an elevated fasting plasma glucose concentration between 100 and $126 \mathrm{mg} / \mathrm{dL}$ [19]. Normal or lean subjects were defined as those with a BMI $<23 \mathrm{~kg} / \mathrm{m}^{2}$ and overweight and obese subjects were defined as those with a BMI $\geq$ $23 \mathrm{~kg} / \mathrm{m}^{2}$ [20]. Ultrasonography with Aloka SSD 4000 and 5000 and Philips HD15 was used to diagnose fatty liver disease according to the practice guideline of the American Gastroenterological Association [21]. The FLI was calculated using the following formula: $\mathrm{FLI}=(e 0.953 *$ loge $(\mathrm{TG})+0.139$ $* \mathrm{BMI}+0.718 *$ loge $(\mathrm{GGT})+0.053 * \mathrm{WC}-15.745) /(1+$ e $0.953 *$ loge $(\mathrm{TG})+0.139 * \mathrm{BMI}+0.718 *$ loge $(\mathrm{GGT})+$ $0.053 * \mathrm{WC}-15.745) * 100$ [4]. The LAP was calculated using the following formula: LAP $=$ (waist circumference $(\mathrm{cm})-65) \times$ triglycerides $(\mathrm{mmol} / \mathrm{L})$ for men and LAP $=$ (waist circumference $(\mathrm{cm})-58) \times$ triglycerides $(\mathrm{mmol} / \mathrm{L})$ for women [12].

This study followed the standards of the Declaration of Helsinki and was approved by the Institutional Review Board of Taipei Veterans General Hospital.

2.2. Biochemical and Serological Markers. Venous blood samples were collected after an overnight fast. Serum HBV surface antigen was tested by radioimmunoassay (Abbott Laboratories, North Chicago, IL, USA), and HCV antibodies were tested by a second-generation enzyme immunoassay. The serum biochemical markers were measured with a Roche/Hitachi Modular Analytics System (Roche Diagnostics GmbH, Mannheim, Germany). 
TABLE 1: Characteristics of subjects with and without metabolic syndrome.

\begin{tabular}{|c|c|c|c|c|}
\hline & $\begin{array}{c}\text { All } \\
(n=29,797)\end{array}$ & $\begin{array}{l}\text { With metabolic syndrome } \\
\qquad(n=8564)\end{array}$ & $\begin{array}{l}\text { Without metabolic syndrome } \\
\qquad(n=21,233)\end{array}$ & $P$ value \\
\hline BMI, $\mathrm{kg} / \mathrm{m}^{2 *}$ & $23.81 \pm 3.58$ & $26.23 \pm 3.31$ & $22.84 \pm 3.21$ & $<0.001$ \\
\hline Age, years* & $52.2 \pm 13.3$ & $56.3 \pm 12.5$ & $50.6 \pm 13.2$ & $<0.001$ \\
\hline $\operatorname{Sex}(M / F)(\%)$ & $\begin{array}{c}16,098 / 13,699 \\
(54.0 / 46.0)\end{array}$ & $\begin{array}{l}6525 / 2039 \\
(76.2 / 23.8)\end{array}$ & $\begin{array}{c}9573 / 11,660 \\
(45.1 / 54.9)\end{array}$ & $<0.001$ \\
\hline $\mathrm{WC}, \mathrm{cm}^{*}$ & $83.8 \pm 10.3$ & $91.5 \pm 8.4$ & $80.7 \pm 9.3$ & $<0.001$ \\
\hline SBP, mmHg & $124.3 \pm 18.6$ & $134.9 \pm 17.2$ & $120.0 \pm 17.4$ & $<0.001$ \\
\hline DBP, mmHg* & $77.5 \pm 14.3$ & $83.7 \pm 17.5$ & $75.0 \pm 11.8$ & $<0.001$ \\
\hline Fasting glucose, $\mathrm{mg} / \mathrm{dL}^{*}$ & $95.5 \pm 24.8$ & $110.4 \pm 35.3$ & $89.5 \pm 15.3$ & $<0.001$ \\
\hline Cholesterol, mg/dL ${ }^{*}$ & $199.2 \pm 37.0$ & $203.1 \pm 38.1$ & $197.6 \pm 36.5$ & $<0.001$ \\
\hline $\mathrm{HDL}, \mathrm{mg} / \mathrm{dL}^{*}$ & $53.7 \pm 15.0$ & $42.9 \pm 10.12$ & $58.0 \pm 14.5$ & $<0.001$ \\
\hline $\mathrm{LDL}, \mathrm{mg} / \mathrm{dL}^{*}$ & $125.3 \pm 32.9$ & $129.0 \pm 33.3$ & $123.8 \pm 32.6$ & $<0.001$ \\
\hline $\mathrm{TG}, \mathrm{mg} / \mathrm{dL}^{*}$ & $130.4 \pm 88.1$ & $201.3 \pm 111.5$ & $101.8 \pm 55.1$ & $<0.001$ \\
\hline AST, IU/L* & $23.1 \pm 13.2$ & $26.0 \pm 18.2$ & $21.9 \pm 10.3$ & $<0.001$ \\
\hline ALT, IU/L* & $27.0 \pm 22.2$ & $35.1 \pm 29.1$ & $23.8 \pm 17.6$ & $<0.001$ \\
\hline GGT, IU/L* & $24.8 \pm 36.8$ & $34.2 \pm 50.9$ & $21.0 \pm 28.4$ & $<0.001$ \\
\hline Platelet, $1000 / \mathrm{mm}^{3 *}$ & $249.8 \pm 60.3$ & $247.5 \pm 62.0$ & $250.8 \pm 59.6$ & $<0.001$ \\
\hline Fatty liver (yes/no) (\%) & $\begin{array}{c}13,255 / 16,542 \\
(44.5 / 55.5)\end{array}$ & $\begin{array}{l}6295 / 2269 \\
(73.5 / 26.5)\end{array}$ & $\begin{array}{c}6960 / 14,273 \\
(32.8 / 67.2)\end{array}$ & $<0.001$ \\
\hline FLI & $27.24 \pm 24.18$ & $50.22 \pm 22.81$ & $17.97 \pm 17.65$ & $<0.001$ \\
\hline LAP & $35.28 \pm 33.59$ & $64.22 \pm 43.52$ & $23.61 \pm 18.57$ & $<0.001$ \\
\hline
\end{tabular}

${ }^{*}$ Expressed as mean \pm standard deviation.

BMI, body mass index; M, male; F, female; WC, waist circumference; SBP, systolic blood pressure; DBP, diastolic blood pressure; HDL, high-density lipoprotein; LDL, low-density lipoprotein; TG, triglyceride; AST, aspartate aminotransferase; ALT, alanine aminotransferase; GGT, gamma-glutamyl transferase; FLI, fatty liver index; LAP, lipid accumulation product.

2.3. Statistical Analysis. The study cohort was first divided by metabolic syndrome, and subjects without ultrasonographic fatty liver disease were selected for further analysis. Pearson's chi-squared test and Student's $t$-test were performed to compare categorical and continuous variables with two samples, respectively. Variables with statistical significance $(P<0.05)$ or proximate to it $(P<0.1)$ in univariate analysis were further included in the multivariate analysis using a logistic regression model with the forward stepwise selection procedure. The ability of serum markers to detect ultrasonographic fatty liver disease was examined using the area under the receiver operator characteristic (AUROC) curves. A $P$ value less than 0.05 was considered to be statistically significant. All statistical analyses were performed using SPSS 17.0 for Windows (SPSS Inc., Chicago, IL, USA).

\section{Results}

3.1. Subject Characteristics Stratified by Metabolic Syndrome. The demographic data of all subjects are summarized in Table 1 . The mean age of the population was 52.2 years and $54 \%$ was male. Metabolic syndrome was diagnosed in $28.7 \%$ of the population. Subjects with metabolic syndrome tended to be older in age, be male, have a higher BMI, serum total cholesterol, low-density lipoprotein cholesterol (LDL-c), alanine aminotransferase (ALT), aspartate aminotransferase (AST), GGT, and fatty liver prevalence, and have lower platelet counts. The FLI averages of subjects with and without metabolic syndrome were 50.2 and 18.0, respectively, while the LAP averages in subjects with and without metabolic syndrome were 64.2 and 23.6, respectively.

We performed a correlation test between total cholesterol and LDL and between ALT and AST. The result showed that the correlation between the sets of data was very high $\left(r^{2}=\right.$ 0.841 and 0.659 , resp.). As the collinearity could affect the calculation of individual predictors even though the whole bundle of predictors could still predict the outcome well, we included only total cholesterol and ALT but not LDL and AST in the multivariate analysis to avoid the condition.

By multivariate analysis, ultrasonographic fatty liver disease and male sex were strongly associated with metabolic syndrome (odds ratio: 2.499 and 3.005, resp.), while higher BMI, older age, and higher ALT and GGT were also associated with metabolic syndrome (Table 2 ). After subjects were divided by ultrasonographic fatty liver disease, the presence of ultrasonographic fatty liver disease was strongly associated with metabolic syndrome as shown in Figure 2(a). We further stratified subjects by age and sex, and the result revealed that male subjects had higher prevalence of metabolic syndrome, and the prevalence of metabolic syndrome increased with age (Figure 2(b)).

3.2. Validation of the FLI and LAP for Identifying Metabolic Syndrome. The discriminative ability of the FLI and LAP to 
TABLE 2: Factors associated with metabolic syndrome by multivariate analysis.

\begin{tabular}{|c|c|c|c|}
\hline & Odds ratio & 95\% confidence interval & $P$ value \\
\hline \multicolumn{4}{|l|}{ All subjects } \\
\hline BMI & 1.272 & $1.258-1.285$ & $<0.001$ \\
\hline Age & 1.041 & $1.038-1.043$ & $<0.001$ \\
\hline ALT & 1.006 & $1.005-1.008$ & $<0.001$ \\
\hline GGT & 1.005 & $1.004-1.006$ & $<0.001$ \\
\hline Platelet & 1.002 & $1.001-1.002$ & $<0.001$ \\
\hline Fatty liver & 2.499 & $2.339-2.670$ & $<0.001$ \\
\hline Male gender & 3.005 & $2.811-3.214$ & $<0.001$ \\
\hline \multicolumn{4}{|l|}{ Females } \\
\hline BMI & 1.311 & $1.288-1.335$ & $<0.001$ \\
\hline Age & 1.065 & $1.059-1.071$ & $<0.001$ \\
\hline ALT & 1.007 & $1.004-1.009$ & $<0.001$ \\
\hline GGT & 1.004 & $1.002-1.005$ & $<0.001$ \\
\hline Platelet & 1.001 & $1.001-1.002$ & 0.002 \\
\hline Fatty liver & 3.275 & $2.888-3.714$ & $<0.001$ \\
\hline \multicolumn{4}{|l|}{ Males } \\
\hline BMI & 1.242 & $1.225-1.259$ & $<0.001$ \\
\hline Age & 1.032 & $1.029-1.035$ & $<0.001$ \\
\hline ALT & 1.006 & $1.004-1.008$ & $<0.001$ \\
\hline GGT & 1.005 & $1.004-1.007$ & $<0.001$ \\
\hline Platelet & 1.002 & $1.001-1.002$ & $<0.001$ \\
\hline Fatty liver & 2.205 & $2.040-2.384$ & $<0.001$ \\
\hline
\end{tabular}

BMI, body mass index; ALT, alanine aminotransferase; GGT, gamma-glutamyl transferase.

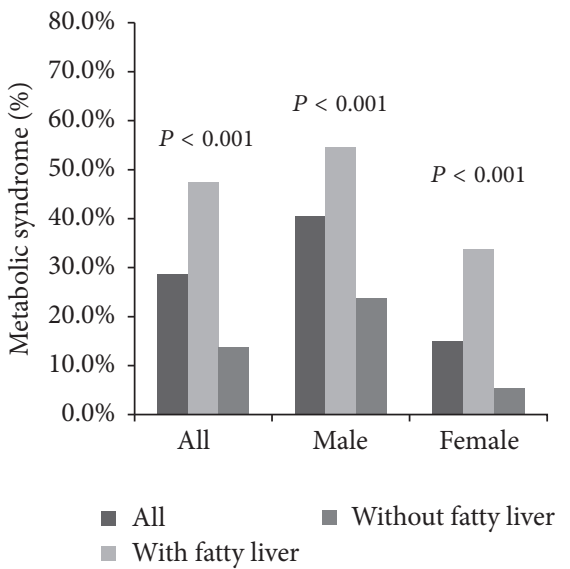

(a)

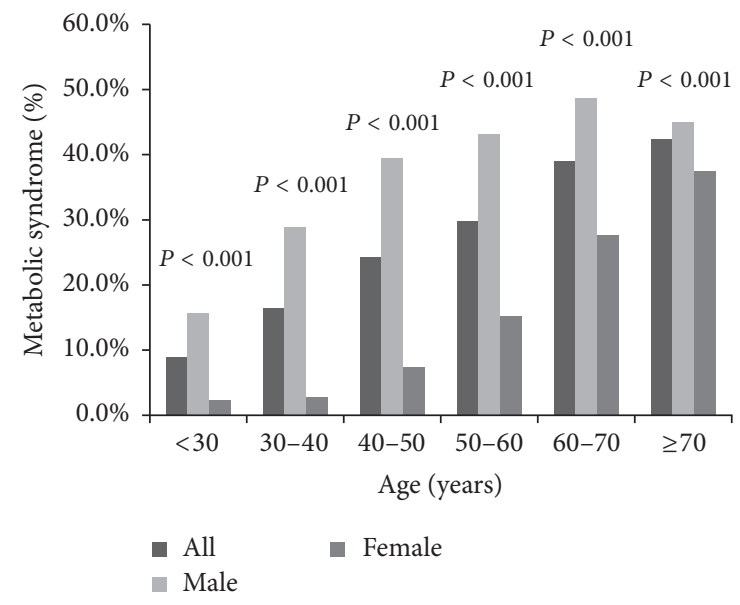

(b)

FIGURE 2: (a) The prevalence of metabolic syndrome divided by the status of fatty liver. (b) The prevalence of metabolic syndrome stratified by age and gender.

identify metabolic syndrome was determined by comparing their AUROC values. The AUROC curve values of the LAP and FLI for the prediction of metabolic syndrome were 0.884 and 0.875 , respectively (Table 3 ). After the subjects were stratified by sex, the AUROC curve values of the LAP and FLI were 0.927 and 0.916 , respectively, in women and 0.856 and 0.818 , respectively, in men. These values were higher than those of other variables such as BMI, fasting glucose,
ALT, GGT, and TG to predict the presence of the metabolic syndrome.

\subsection{Subject Characteristics Stratified by the IFG and Validation} of the FLI and LAP for Identifying IFG. IFG was diagnosed in $21.8 \%$ of the population. Subjects with IFG had similar characteristics to those with metabolic syndrome. They tended to be older in age, be male, have a higher BMI, ALT, GGT, and 
TABLE 3: Comparison of AUROC curve values among noninvasive markers for predicting metabolic syndrome.

\begin{tabular}{|c|c|c|c|c|}
\hline & AUROC & 95\% confidence interval & Standard error & $P$ value \\
\hline \multicolumn{5}{|l|}{ All subjects } \\
\hline LAP & 0.884 & $0.893-0.901$ & 0.002 & $<0.001$ \\
\hline FLI & 0.875 & $0.871-0.879$ & 0.002 & $<0.001$ \\
\hline TG & 0.853 & $0.849-0.858$ & 0.002 & $<0.001$ \\
\hline HDL & 0.820 & $0.815-0.825$ & 0.003 & $<0.001$ \\
\hline WC & 0.817 & $0.812-0.821$ & 0.002 & $<0.001$ \\
\hline BMI & 0.785 & $0.779-0.790$ & 0.003 & $<0.001$ \\
\hline Fasting glucose & 0.775 & $0.769-0.781$ & 0.003 & $<0.001$ \\
\hline GGT & 0.724 & $0.717-0.730$ & 0.003 & $<0.001$ \\
\hline Fatty liver & 0.704 & $0.697-0.710$ & 0.003 & $<0.001$ \\
\hline ALT & 0.691 & $0.684-0.698$ & 0.003 & $<0.001$ \\
\hline LDL & 0.548 & $0.541-0.556$ & 0.004 & $<0.001$ \\
\hline Cholesterol & 0.543 & $0.536-0.550$ & 0.004 & $<0.001$ \\
\hline \multicolumn{5}{|l|}{ Female subjects } \\
\hline LAP & 0.927 & $0.922-0.933$ & 0.003 & $<0.001$ \\
\hline FLI & 0.916 & $0.910-0.922$ & 0.003 & $<0.001$ \\
\hline TG & 0.874 & $0.866-0.882$ & 0.004 & $<0.001$ \\
\hline WC & 0.853 & $0.845-0.862$ & 0.004 & $<0.001$ \\
\hline Fasting glucose & 0.850 & $0.840-0.860$ & 0.005 & $<0.001$ \\
\hline BMI & 0.833 & $0.824-0.842$ & 0.005 & $<0.001$ \\
\hline HDL & 0.825 & $0.816-0.835$ & 0.005 & $<0.001$ \\
\hline Fatty liver & 0.747 & $0.736-0.759$ & 0.006 & $<0.001$ \\
\hline GGT & 0.733 & $0.721-0.744$ & 0.006 & $<0.001$ \\
\hline ALT & 0.693 & $0.681-0.706$ & 0.006 & $<0.001$ \\
\hline LDL & 0.591 & $0.577-0.604$ & 0.007 & $<0.001$ \\
\hline Cholesterol & 0.575 & $0.562-0.589$ & 0.007 & $<0.001$ \\
\hline \multicolumn{5}{|l|}{ Male subjects } \\
\hline LAP & 0.856 & $0.850-0.862$ & 0.003 & $<0.001$ \\
\hline TG & 0.825 & $0.818-0.831$ & 0.003 & $<0.001$ \\
\hline FLI & 0.818 & $0.812-0.825$ & 0.003 & $<0.001$ \\
\hline $\mathrm{HDL}$ & 0.780 & $0.773-0.788$ & 0.004 & $<0.001$ \\
\hline Fasting glucose & 0.744 & $0.736-0.752$ & 0.004 & $<0.001$ \\
\hline WC & 0.741 & $0.733-0.748$ & 0.004 & $<0.001$ \\
\hline BMI & 0.723 & $0.716-0.731$ & 0.004 & $<0.001$ \\
\hline GGT & 0.659 & $0.650-0.667$ & 0.004 & $<0.001$ \\
\hline ALT & 0.634 & $0.625-0.643$ & 0.004 & $<0.001$ \\
\hline Fatty liver & 0.658 & $0.650-0.667$ & 0.004 & $<0.001$ \\
\hline Cholesterol & 0.542 & $0.533-0.551$ & 0.005 & $<0.001$ \\
\hline $\mathrm{LDL}$ & 0.518 & $0.508-0.527$ & 0.005 & $<0.001$ \\
\hline
\end{tabular}

AUROC, area under the receiver operating characteristic; LAP, lipid accumulation product; FLI, fatty liver index; TG, triglyceride; HDL, high-density lipoprotein; WC, waist circumference; BMI, body mass index; GGT, gamma-glutamyl transferase; ALT: alanine aminotransferase; LDL, low-density lipoprotein.

fatty liver prevalence, and have lower platelet counts. The FLI averages of subjects with and without IFG were 38.15 and 23.3, respectively, while the LAP averages were 46.51 and 30.69 , respectively (Table 4). The discriminative ability of FLI and LAP to identify IFG was better in subjects without fatty liver disease ( 0.669 and 0.643 , respectively) than in subjects with fatty liver disease. The FLI and LAP also predicted IFG in subjects with lower BMI (0.673 and 0.642, resp.) (Table 5).
3.4. Characteristics of Subjects without Ultrasonographic Fatty Liver Disease Stratified by Metabolic Syndrome. The demographics of subjects without ultrasonographic fatty liver disease are summarized in Table 6 . The average age of the subjects was 50.9 years and $45 \%$ were male. Subjects with metabolic syndrome tended to be older in age, be male, have higher BMI, ALT, and GGT, and have lower platelet counts. The FLI and LAP were 37 and 47, respectively, in subjects with 
TABLE 4: Characteristics of subjects with IFG.

\begin{tabular}{|c|c|c|c|}
\hline & $\begin{array}{c}\text { IFG } \\
(n=5001)\end{array}$ & $\begin{array}{c}\text { Non-IFG } \\
(n=22,970)\end{array}$ & $P$ value \\
\hline BMI, $\mathrm{kg} / \mathrm{m}^{2 *}$ & $25.28 \pm 3.48$ & $23.34 \pm 3.44$ & $<0.001$ \\
\hline Age, years* & $57.7 \pm 11.8$ & $50.3 \pm 13.1$ & $<0.001$ \\
\hline $\operatorname{Sex}(M / F)(\%)$ & $\begin{array}{l}3036 / 1965 \\
(60.7 / 39.3)\end{array}$ & $\begin{array}{c}11,859 / 11,111 \\
(51.6 / 48.4)\end{array}$ & $<0.001$ \\
\hline $\mathrm{WC}, \mathrm{cm}^{*}$ & $88.2 \pm 9.4$ & $82.3 \pm 9.9$ & $<0.001$ \\
\hline SBP, mmHg ${ }^{*}$ & $131.8 \pm 18.5$ & $121.7 \pm 17.8$ & $<0.001$ \\
\hline DBP, $\mathrm{mmHg}^{*}$ & $81.2 \pm 15.5$ & $76.4 \pm 13.3$ & $<0.001$ \\
\hline Fasting glucose, $\mathrm{mg} / \mathrm{dL}^{*}$ & $107.6 \pm 6.8$ & $87 \pm 7.6$ & $<0.001$ \\
\hline Cholesterol, mg/dL ${ }^{*}$ & $203.8 \pm 37.6$ & $197.9 \pm 36.5$ & $<0.001$ \\
\hline $\mathrm{HDL}, \mathrm{mg} / \mathrm{dL}^{*}$ & $50.4 \pm 13.4$ & $55 \pm 15.3$ & $<0.001$ \\
\hline $\mathrm{LDL}, \mathrm{mg} / \mathrm{dL}^{*}$ & $129 \pm 32.7$ & $124.4 \pm 32.7$ & $<0.001$ \\
\hline $\mathrm{TG}, \mathrm{mg} / \mathrm{dL}^{*}$ & $152.3 \pm 90$ & $120.9 \pm 80.8$ & $<0.001$ \\
\hline AST, IU/L* ${ }^{*}$ & $25.2 \pm 19$ & $22.3 \pm 10.5$ & $<0.001$ \\
\hline ALT, IU/L* ${ }^{*}$ & $31.6 \pm 28.9$ & $25.4 \pm 19.2$ & $<0.001$ \\
\hline GGT, IU/L* & $30.7 \pm 51.4$ & $22.4 \pm 27.3$ & $<0.001$ \\
\hline Platelet, $1000 / \mathrm{mm}^{3 *}$ & $245.3 \pm 60.1$ & $251.4 \pm 59.9$ & $<0.001$ \\
\hline Fatty liver (yes/no) (\%) & $\begin{array}{l}3150 / 1851 \\
(63.0 / 37.0)\end{array}$ & $\begin{array}{c}8737 / 14,233 \\
(38.0 / 62.0)\end{array}$ & $<0.001$ \\
\hline FLI & $38.15 \pm 25.02$ & $23.3 \pm 22.28$ & $<0.001$ \\
\hline LAP & $46.51 \pm 35.15$ & $30.69 \pm 29.6$ & $<0.001$ \\
\hline
\end{tabular}

${ }^{*}$ Expressed as mean \pm standard deviation.

IFG, impaired fasting glucose; BMI, body mass index; M, male; F, female; WC, waist circumference; SBP, systolic blood pressure; DBP, diastolic blood pressure; HDL, high-density lipoprotein; LDL, low-density lipoprotein; TG, triglyceride; AST, aspartate aminotransferase; ALT, alanine aminotransferase; GGT, gammaglutamyl transferase; LAP, lipid accumulation product.

TABLE 5: Comparison of AUROC curve values among noninvasive markers for predicting IFG.

\begin{tabular}{lccc}
\hline & AUROC & $95 \%$ confidence interval & Standard error \\
\hline All & & & $P$ value \\
FLI & 0.689 & 0.004 & $0.682-0.697$ \\
LAP & 0.675 & 0.004 & $0.667-0.683$ \\
With fatty liver & & & $<0.001$ \\
FLI & 0.609 & 0.006 & $0.598-0.62$ \\
LAP & 0.602 & 0.006 & $0.591-0.613$ \\
Without fatty liver & & & $<0.001$ \\
FLI & 0.669 & 0.006 & $0.656-0.681$ \\
LAP & 0.643 & 0.007 & $0.629-0.656$ \\
BMI (lean and normal) & & & $<0.001$ \\
FLI & 0.673 & 0.008 & $0.658-0.688$ \\
LAP & 0.642 & 0.008 & $0.626-0.659$ \\
BMI (overweight and obesity) & & & $<0.001$ \\
FLI & 0.616 & 0.005 & $<0.001$ \\
LAP & 0.605 & 0.005 & $<0.001$ \\
\hline
\end{tabular}

IFG, impaired fasting glucose; AUROC, area under the receiver operating characteristic; FLI, fatty liver index; LAP, lipid accumulation product; BMI, body mass index.

metabolic syndrome and 12 and 18, respectively, in subjects without metabolic syndrome. By multivariate analysis, older age, male sex, and higher BMI, ALT, and GGT were still associated with metabolic syndrome in subjects without fatty liver disease (Table 7). We further stratified subjects by age and sex, and the result revealed that male subjects had higher prevalence of metabolic syndrome, and the prevalence of metabolic syndrome increased with age (Figure 3 ). 
TABLE 6: Characteristics of non-fatty liver subjects with and without metabolic syndrome.

\begin{tabular}{|c|c|c|c|c|}
\hline & $\begin{array}{c}\text { All } \\
(n=16,542)\end{array}$ & $\begin{array}{l}\text { With metabolic syndrome } \\
\qquad(n=2269)\end{array}$ & $\begin{array}{l}\text { Without metabolic syndrome } \\
\qquad(n=14,273)\end{array}$ & $P$ value \\
\hline BMI, $\mathrm{kg} / \mathrm{m}^{2 *}$ & $22.32 \pm 2.90$ & $24.79 \pm 2.72$ & $21.93 \pm 2.73$ & $<0.001$ \\
\hline Age, years* & $50.9 \pm 14.1$ & $58.6 \pm 13.8$ & $49.6 \pm 13.8$ & $<0.001$ \\
\hline $\operatorname{Sex}(M / F)(\%)$ & $\begin{array}{l}7388 / 9154 \\
(44.7 / 55.3)\end{array}$ & $\begin{array}{c}1765 / 504 \\
(77.8 / 22.2)\end{array}$ & $\begin{array}{l}5623 / 8650 \\
(39.4 / 60.6)\end{array}$ & $<0.001$ \\
\hline $\mathrm{WC}, \mathrm{cm}^{*}$ & $79.5 \pm 8.9$ & $88.4 \pm 7.3$ & $78.1 \pm 8.4$ & $<0.001$ \\
\hline SBP, mmHg ${ }^{*}$ & $121.1 \pm 18.5$ & $136.0 \pm 17.6$ & $118.8 \pm 17.6$ & $<0.001$ \\
\hline DBP, $\mathrm{mmHg}^{*}$ & $75.3 \pm 11.6$ & $82.6 \pm 11.1$ & $74.1 \pm 11.3$ & $<0.001$ \\
\hline Fasting glucose, $\mathrm{mg} / \mathrm{dL}^{*}$ & $90.7 \pm 19.1$ & $107.1 \pm 32.7$ & $88.1 \pm 14.2$ & $<0.001$ \\
\hline Cholesterol, mg/dL ${ }^{*}$ & $194.5 \pm 36.0$ & $195.8 \pm 37.7$ & $194.3 \pm 35.7$ & 0.058 \\
\hline $\mathrm{HDL}, \mathrm{mg} / \mathrm{dL}^{*}$ & $58.2 \pm 15.5$ & $44.2 \pm 11.4$ & $60.4 \pm 14.9$ & $<0.001$ \\
\hline $\mathrm{LDL}, \mathrm{mg} / \mathrm{dL}^{*}$ & $120.5 \pm 31.7$ & $124.2 \pm 32.3$ & $119.9 \pm 31.6$ & $<0.001$ \\
\hline $\mathrm{TG}, \mathrm{mg} / \mathrm{dL}^{*}$ & $101.0 \pm 57.2$ & $168.0 \pm 81.5$ & $90.3 \pm 43.7$ & $<0.001$ \\
\hline AST, IU/L* & $21.1 \pm 11.4$ & $23.1 \pm 23.9$ & $20.8 \pm 7.7$ & $<0.001$ \\
\hline ALT, IU/L* & $21.2 \pm 17.2$ & $26.2 \pm 33.2$ & $20.4 \pm 12.7$ & $<0.001$ \\
\hline GGT, IU/L* & $20.0 \pm 33.9$ & $30.4 \pm 66.9$ & $18.3 \pm 24.5$ & $<0.001$ \\
\hline Platelet, $1000 / \mathrm{mm}^{3 *}$ & $247.6 \pm 60.9$ & $238.7 \pm 65.5$ & $249.0 \pm 60.0$ & $<0.001$ \\
\hline FLI & $15.61 \pm 16.27$ & $36.99 \pm 20.27$ & $12.21 \pm 12.54$ & $<0.001$ \\
\hline LAP & $22.35 \pm 18.81$ & $47.10 \pm 27.58$ & $18.41 \pm 13.27$ & $<0.001$ \\
\hline
\end{tabular}

*Expressed as mean \pm standard deviation.

BMI, body mass index; M, male; F, female; WC, waist circumference; SBP, systolic blood pressure; DBP, diastolic blood pressure; HDL, high-density lipoprotein; LDL, low-density lipoprotein; TG, triglyceride; AST, aspartate aminotransferase; ALT, alanine aminotransferase; GGT, gamma-glutamyl transferase; FLI, fatty liver index; LAP, lipid accumulation product.

TABLE 7: Risk factors of metabolic syndrome in subjects with non-fatty liver disease by multivariate analysis.

\begin{tabular}{lccc}
\hline & Odds ratio & $95 \%$ confidence interval & $P$ value \\
\hline All subjects & & & $<0.001$ \\
BMI & 1.371 & $1.346-1.397$ & $<0.001$ \\
Age & 1.042 & $1.038-1.046$ & $<0.001$ \\
ALT & 1.007 & $1.004-1.010$ & $<0.001$ \\
GGT & 1.004 & $1.002-1.006$ & 0.001 \\
Platelet & 1.001 & $1.001-1.002$ & $<0.001$ \\
Male gender & 4.071 & $3.628-4.568$ & \\
Female subjects & & & $<0.001$ \\
BMI & 1.382 & $1.339-1.426$ & $<0.001$ \\
Age & 1.080 & $1.070-1.089$ & $<0.001$ \\
ALT & 1.010 & $1.005-1.015$ & 0.028 \\
Platelet & 1.002 & $1.000-1.003$ & \\
Male subjects & & $1.320-1.383$ & $<0.001$ \\
BMI & 1.351 & $1.028-1.037$ & $<0.001$ \\
Age & 1.032 & $1.001-1.009$ & 0.021 \\
ALT & 1.005 & $1.003-1.007$ & $1.001-1.003$ \\
GGT & 1.005 & & $<0.001$ \\
Platelet & 1.002 & 0.002 \\
\hline
\end{tabular}

BMI, body mass index; ALT, alanine aminotransferase; GGT, gamma-glutamyl transferase.

3.5. Validation of the FLI and LAP for Identifying Metabolic Syndrome in Subjects without Ultrasonographic Fatty Liver Disease. The predictive ability of FLI and LAP to identify metabolic syndrome was determined by comparing their
AUROC curve values (Table 8). The AUROC curve values of the LAP and FLI to predict the presence of metabolic syndrome were 0.871 and 0.879 , respectively. After the subjects were stratified by sex, the AUROC curve values of the LAP 
TABLE 8: Comparison of AUROC curve values among non-fatty liver subjects with metabolic syndrome.

\begin{tabular}{|c|c|c|c|c|}
\hline & AUROC & 95\% confidence interval & Standard error & $P$ value \\
\hline \multicolumn{5}{|l|}{ All subjects } \\
\hline FLI & 0.879 & $0.872-0.885$ & 0.003 & $<0.001$ \\
\hline LAP & 0.871 & $0.863-0.879$ & 0.004 & $<0.001$ \\
\hline Triglyceride & 0.828 & $0.818-0.838$ & 0.005 & $<0.001$ \\
\hline WC & 0.827 & $0.819-0.835$ & 0.004 & $<0.001$ \\
\hline HDL & 0.822 & $0.832-0.813$ & 0.005 & $<0.001$ \\
\hline BMI & 0.783 & $0.774-0.792$ & 0.005 & $<0.001$ \\
\hline Fasting glucose & 0.774 & $0.762-0.785$ & 0.006 & $<0.001$ \\
\hline GGT & 0.693 & $0.682-0.704$ & 0.006 & $<0.001$ \\
\hline Age & 0.679 & $0.667-0.691$ & 0.006 & $<0.001$ \\
\hline ALT & 0.619 & $0.607-0.631$ & 0.006 & $<0.001$ \\
\hline LDL & 0.543 & $0.531-0.556$ & 0.006 & $<0.001$ \\
\hline Cholesterol & 0.513 & $0.501-0.526$ & 0.007 & 0.04 \\
\hline \multicolumn{5}{|l|}{ Female subjects } \\
\hline LAP & 0.921 & $0.909-0.932$ & 0.006 & $<0.001$ \\
\hline FLI & 0.909 & $0.898-0.921$ & 0.006 & $<0.001$ \\
\hline Triglyceride & 0.860 & $0.842-0.878$ & 0.009 & $<0.001$ \\
\hline Fasting glucose & 0.841 & $0.820-0.862$ & 0.011 & $<0.001$ \\
\hline WC & 0.830 & $0.811-0.849$ & 0.010 & $<0.001$ \\
\hline HDL & 0.822 & $0.841-0.803$ & 0.010 & $<0.001$ \\
\hline BMI & 0.812 & $0.792-0.831$ & 0.010 & $<0.001$ \\
\hline Age & 0.784 & $0.765-0.804$ & 0.010 & $<0.001$ \\
\hline GGT & 0.673 & $0.650-0.697$ & 0.012 & $<0.001$ \\
\hline ALT & 0.589 & $0.563-0.615$ & 0.013 & $<0.001$ \\
\hline LDL & 0.574 & $0.548-0.600$ & 0.013 & $<0.001$ \\
\hline Cholesterol & 0.547 & $0.520-0.574$ & 0.014 & $<0.001$ \\
\hline \multicolumn{5}{|l|}{ Male subjects } \\
\hline LAP & 0.844 & $0.834-0.855$ & 0.005 & $<0.001$ \\
\hline FLI & 0.814 & $0.803-0.825$ & 0.005 & $<0.001$ \\
\hline Triglyceride & 0.787 & $0.774-0.801$ & 0.007 & $<0.001$ \\
\hline $\mathrm{HDL}$ & 0.772 & $0.786-0.759$ & 0.007 & $<0.001$ \\
\hline WC & 0.757 & $0.745-0.768$ & 0.006 & $<0.001$ \\
\hline Fasting glucose & 0.738 & $0.724-0.753$ & 0.007 & $<0.001$ \\
\hline BMI & 0.723 & $0.711-0.736$ & 0.006 & $<0.001$ \\
\hline Age & 0.615 & $0.601-0.630$ & 0.007 & $<0.001$ \\
\hline GGT & 0.615 & $0.600-0.630$ & 0.008 & $<0.001$ \\
\hline ALT & 0.559 & $0.544-0.575$ & 0.008 & $<0.001$ \\
\hline Cholesterol & 0.517 & $0.502-0.533$ & 0.008 & 0.029 \\
\hline LDL & 0.514 & $0.499-0.529$ & 0.008 & 0.074 \\
\hline
\end{tabular}

AUROC, area under the receiver operating characteristic; FLI, fatty liver index; LAP, lipid accumulation product; TG, triglyceride; WC, waist circumference; HDL, high-density lipoprotein; BMI, body mass index; GGT, gamma-glutamyl transferase; ALT, alanine aminotransferase; LDL, low-density lipoprotein.

and FLI were 0.921 and 0.909 , respectively, in women and 0.844 and 0.814 , respectively, in men.

\section{Discussion}

The FLI and LAP are indexes originally designed to assess the risk of fatty liver and cardiovascular disease, respectively, and both have been shown to be good markers of metabolic syndrome $[4,10,12,13]$. In the present study, fatty liver disease was closely associated with metabolic syndrome, and both the FLI and LAP were predictive of metabolic syndrome. For people without fatty liver, both indexes were still strong predictors of metabolic syndrome.

Twenty-seven percent of the population aged more than 25 years in the US [22] and approximately 12\% between 1999 and 2002 in Taiwan have been reported to have metabolic 


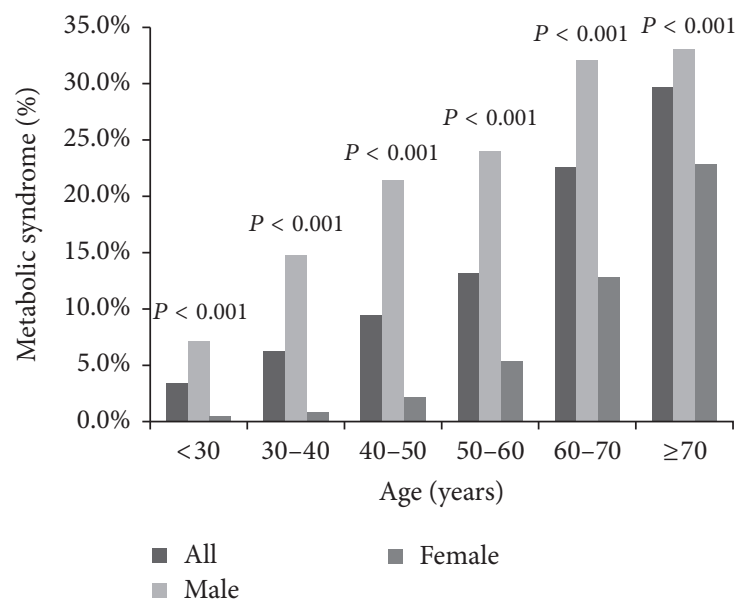

FIgURE 3: The prevalence of metabolic syndrome in subjects without sonographic fatty liver stratified by age and gender.

syndrome [23]. However, the prevalence of metabolic syndrome was much higher in the present study $(28 \%)$, which may be because of the westernization of diet, greater awareness of the syndrome, or higher socioeconomic status of the subjects. It was noteworthy that the proportion of subjects with metabolic syndrome increased with age and the trend existed in both sexes (Figure 2(b)).

The prevalence of NAFLD, which varies by the diagnostic modality and ethnicity, ranges from $23 \%$ to $51 \%$ in Asian populations, and the prevalence of fatty liver disease in this study population falls within this range at $44.5 \%$ [24, 25]. Fatty liver disease was the strongest factor associated with metabolic syndrome in both sexes (Table 2 and Figure 2(a)). However, metabolic syndrome also exists in subjects without fatty liver disease, a group of subjects who have cardiovascular risk but is rarely focused on. Han et al. observed that $12 \%$ of subjects without ultrasonographic fatty liver disease at the end of the study developed metabolic syndrome in South Korea [26]. Up to $26.5 \%$ of subjects with mild or absent liver steatosis were also noted to have metabolic syndrome in Italy [27]. Metabolic syndrome can even occur in children without fatty liver disease. Schwimmer et al. defined the absence of NAFLD as the combination of a normal ALT level $(<30 \mathrm{U} / \mathrm{L})$ and the absence of hepatomegaly and found that metabolic syndrome exists in $15 \%$ of children with a mean age of 12.7 years without NAFLD [28]. After subjects with ultrasonographic fatty liver disease were excluded, the prevalence of metabolic syndrome in our study fell to $13.7 \%$, similar to that in previous studies. Besides, an age-related increasing trend in the prevalence of metabolic syndrome was observed in both subjects with and without fatty liver disease (Figure 3).

Prediabetes has been recognized as a cardiometabolic risk factor and its phenotypes have been thoroughly assessed [29]. In this study, we investigated the role of the FLI and LAP to predict IFG after the subjects were stratified by the diagnosis of fatty liver disease and BMI. Interestingly, the results showed that the FLI and LAP had better predictive abilities for lean subjects and those without fatty liver than their counterparts. These findings suggest that the FLI and LAP could help clinical physicians identify a high-risk group of cardiometabolic diseases in these two commonly overlooked populations.

Bedogni et al. developed the FLI as an accurate index which correlates well with ultrasonographic fatty liver disease. The FLI has limited utility for the quantification of hepatic steatosis [30-32], but it has been validated by abdominal ultrasonography in several populations with an AUROC curve between 0.930 and 0.840 in Western countries to identify fatty liver disease, though the accuracy is less prominent in Asian countries probably because of variation of ethnicity, dietary, and environmental factors $[4,33]$.

Furthermore, the FLI is associated with cardiovascular risk factors including hypertension and carotid plaques [9, 34 ] and can predict cardiovascular and liver-related mortality [35-37]. As cardiovascular disease, obesity, NAFLD, and metabolic syndrome are intertwined, Rogulj et al. suggested that the FLI may be an optimal diagnostic method for metabolic syndrome in terms of sensitivity and specificity [10]. Their findings were comparable with those of the present study in that the FLI was better than other variables to predict metabolic syndrome with an AUROC curve of 0.875 . We further analyzed the performance of the FLI to identify metabolic syndrome in subjects without fatty liver disease. Our results showed that the FLI was a reliable tool to predict metabolic syndrome with an AUROC curve of 0.879 . Accordingly, subjects without fatty liver disease and a high FLI may also need an intensified counselling plan.

The LAP, which includes TG concentration and WC, was first proposed by Kahn to recognize cardiovascular risk [14, 38]. The LAP was further noticed to have a strong association with insulin resistance $[39,40]$, glucose dysregulation [40, $41]$, and type $2 \mathrm{DM}[42,43]$ and was also associated with the stroke incidence in a 9.2-year prospective Chinese study [44]. Bedogni et al. concluded that the LAP can be a good marker of liver steatosis and the conclusion was validated in Korea although the accuracy is lower in the Asian population $[12,45]$. Several studies found that the LAP could be a good indicator of metabolic syndrome with an AUROC curve greater than $0.9[13,46-48]$. The present study revealed that the LAP was a useful tool to identify subjects with metabolic syndrome, which was comparable to the findings of previous studies. However, in our analysis, the LAP was found to be a better predictor of metabolic syndrome among women, which was different from the results of another Taiwanese study which found that the LAP was better to recognize metabolic syndrome among men [47]. The inclusion of people aged over 50 years and the small sample size may explain the difference in the results. Furthermore, we also found that the LAP could reliably identify metabolic syndrome (AUROC: 0.879 ) even in subjects without fatty liver, and the predictive power was better among women as well.

TG values already have a high power to predict metabolic syndrome. With the addition of the FLI and LAP, more people with risk of cardiovascular disease can be recognized. The awareness of the risk with further implementation of an intensive counselling plan will be of great importance to prevent cardiovascular disease. 
The large sample size and detailed biochemistry data are the strengths of this study. However, there are several noteworthy limitations. First, the study population had a higher socioeconomic status and subjects could afford the expense of a physical check-up, so the results might not represent the general population. Second, alcohol consumption was not evaluated in the present study. However, the prevalence of alcoholism was surveyed to be $1.5 \%$ in Taiwanese communities [49], and the impact on our results should be small. Third, liver biopsy is the gold standard for the diagnosis of fatty liver disease. However, the invasiveness of that procedure is not justified for surveillance in the general population. The diagnosis of fatty liver disease was made by at least two of three abnormal findings on abdominal ultrasonography: diffusely increased echogenic liver as compared with the kidney or spleen, vascular blurring, and deep attenuation of the ultrasound signal with sensitivity of $89 \%$ and specificity of $93 \%[50,51]$. Fourth, we could not analyze impaired glucose tolerance, another important phenotype of cardiometabolic risk, because the oral glucose tolerance test was not performed in our cohort. Further studies are warranted to elucidate the correlation between the FLI/LAP and cardiometabolic risk.

In conclusion, metabolic syndrome increases risk of cardiovascular disease, and the FLI and LAP could be used to recognize the syndrome in people without fatty liver disease who also require lifestyle modifications and counseling in addition to their counterparts with fatty liver disease.

\section{Competing Interests}

The authors declare that there are no competing interests regarding the publication of this paper.

\section{Acknowledgments}

This study was supported by grants from the National Science Council (NSC 101-2314-B-075-014) and Taipei Veterans General Hospital (V102C-151).

\section{References}

[1] K. G. M. M. Alberti, R. H. Eckel, S. M. Grundy et al., "Harmonizing the metabolic syndrome: a joint interim statement of the international diabetes federation task force on epidemiology and prevention; national heart, lung, and blood institute; American heart association; world heart federation; international atherosclerosis society; and international association for the study of obesity," Circulation, vol. 120, no. 16, pp. 1640-1645, 2009.

[2] G. Musso, R. Gambino, S. Bo et al., "Should nonalcoholic fatty liver disease be included in the definition of metabolic syndrome? A cross-sectional comparison with Adult Treatment Panel III criteria in nonobese nondiabetic subjects," Diabetes Care, vol. 31, no. 3, pp. 562-568, 2008.

[3] P. Almeda-Valdes, D. Cuevas-Ramos, and C. A. Aguilar-Salinas, "Metabolic syndrome and non-alcoholic fatty liver disease," Annals of Hepatology, vol. 8, no. 1, pp. S18-S24, 2009.

[4] G. Bedogni, S. Bellentani, L. Miglioli et al., "The fatty liver index: a simple and accurate predictor of hepatic steatosis in the general population," BMC Gastroenterology, vol. 6, article no. 33, 2006.

[5] C. H. Jung, Y. M. Kang, J. E. Jang et al., "Fatty liver index is a risk determinant of incident type 2 diabetes in a metabolically healthy population with obesity," Obesity, vol. 24, no. 6, pp. 1373-1379, 2016.

[6] S. Jäger, S. Jacobs, J. Kröger et al., "Association between the fatty liver index and risk of type 2 diabetes in the EPIC-Potsdam study," PLoS ONE, vol. 10, no. 4, Article ID e0124749, 2015.

[7] C. H. Jung, W. J. Lee, J. Y. Hwang et al., "Assessment of the fatty liver index as an indicator of hepatic steatosis for predicting incident diabetes independently of insulin resistance in a Korean population," Diabetic Medicine, vol. 30, no. 4, pp. 428435, 2013.

[8] L. Bozkurt, C. S. Göbl, A. Tura et al., "Fatty liver index predicts further metabolic deteriorations in women with previous gestational diabetes," PLoS ONE, vol. 7, no. 2, Article ID e32710, 2012.

[9] J. H. Huh, S. V. Ahn, S. B. Koh et al., "A prospective study of fatty liver index and incident hypertension: the KoGES-ARIRANG study," PLoS ONE, vol. 10, no. 11, Article ID e0143560, 2015.

[10] D. Rogulj, P. Konjevoda, M. Milić, M. Mladinić, and A.M. Domijan, "Fatty liver index as an indicator of metabolic syndrome," Clinical Biochemistry, vol. 45, no. 1-2, pp. 68-71, 2012.

[11] E. Lerchbaum, H.-J. Gruber, V. Schwetz et al., "Fatty liver index in polycystic ovary syndrome," European Journal of Endocrinology, vol. 165, no. 6, pp. 935-943, 2011.

[12] G. Bedogni, H. S. Kahn, S. Bellentani, and C. Tiribelli, "A simple index of lipid overaccumulation is a good marker of liver steatosis," BMC Gastroenterology, vol. 10, article no. 98, 2010.

[13] M. J. Taverna, M. T. Martínez-Larrad, G. D. Frechtel, and M. Serrano-Ríos, "Lipid accumulation product: a powerful marker of metabolic syndrome in healthy population," European Journal of Endocrinology, vol. 164, no. 5, pp. 559-567, 2011.

[14] H. S. Kahn, “The 'lipid accumulation product' performs better than the body mass index for recognizing cardiovascular risk: a population-based comparison," BMC Cardiovascular Disorders, vol. 5, article 26, 2005.

[15] W.-C. Wu, C.-Y. Wu, Y.-J. Wang et al., "Updated thresholds for serum alanine aminotransferase level in a large-scale population study composed of 34346 subjects," Alimentary Pharmacology and Therapeutics, vol. 36, no. 6, pp. 560-568, 2012.

[16] Y.-L. Cheng, Y.-J. Wang, W.-Y. Kao et al., "Inverse association between hepatitis B virus infection and fatty liver disease: a large-scale study in populations seeking for check-up," PLoS ONE, vol. 8, Article ID e72049, 2013.

[17] Y.-L. Cheng, Y.-C. Wang, K.-H. Lan et al., "Anti-hepatitis C virus seropositivity is not associated with metabolic syndrome irrespective of age, gender and fibrosis," Annals of Hepatology, vol. 14, no. 2, pp. 181-189, 2015.

[18] B.-L. Yang, W.-C. Wu, K.-C. Fang et al., "External validation of fatty liver index for identifying ultrasonographic fatty liver in a large-scale cross-sectional study in Taiwan," PLoS ONE, vol. 10, no. 3, Article ID e0120443, 2015.

[19] D. M. Nathan, M. B. Davidson, R. A. DeFronzo et al., "Impaired fasting glucose and impaired glucose tolerance: implications for care," Diabetes Care, vol. 30, no. 3, pp. 753-759, 2007.

[20] W. C. Hsu, M. R. G. Araneta, A. M. Kanaya, J. L. Chiang, and W. Fujimoto, "BMI cut points to identify at-Risk asian americans for type 2 diabetes screening," Diabetes Care, vol. 38, no. 1, pp. 150-158, 2015. 
[21] N. Chalasani, Z. Younossi, J. E. Lavine et al., "The diagnosis and management of non-alcoholic fatty liver disease: practice guideline by the American Gastroenterological Association, American Association for the Study of Liver Diseases, and American College of Gastroenterology," Gastroenterology, vol. 142, no. 7, pp. 1592-1609, 2012.

[22] E. S. Ford, W. H. Giles, and A. H. Mokdad, "Increasing prevalence of the metabolic syndrome among U.S. adults," Diabetes Care, vol. 27, no. 10, pp. 2444-2449, 2004.

[23] C.-F. Jan, C.-J. Chen, Y.-H. Chiu et al., "A population-based study investigating the association between metabolic syndrome and hepatitis B/C infection (Keelung Community-based Integrated Screening study No. 10)," International Journal of Obesity, vol. 30, no. 5, pp. 794-799, 2006.

[24] J. Y. Lee, K. M. Kim, S. G. Lee et al., "Prevalence and risk factors of non-alcoholic fatty liver disease in potential living liver donors in Korea: a review of 589 consecutive liver biopsies in a single center," Journal of Hepatology, vol. 47, no. 2, pp. 239244, 2007.

[25] X.-H. Hou, Y.-X. Zhu, H.-J. Lu et al., "Non-alcoholic fatty liver disease's prevalence and impact on alanine aminotransferase associated with metabolic syndrome in the Chinese," Journal of Gastroenterology and Hepatology (Australia), vol. 26, no. 4, pp. 722-730, 2011.

[26] E. N. Han, E. S. Cheong, J. I. Lee, M. C. Kim, C. D. Byrne, and K. Sung, "Change in fatty liver status and 5-year risk of incident metabolic syndrome: A Retrospective Cohort Study," Clinical Hypertension, vol. 21, no. 1, 2015.

[27] F. Angelico, M. Del Ben, R. Conti et al., "Insulin resistance, the metabolic syndrome, and nonalcoholic fatty liver disease," Journal of Clinical Endocrinology and Metabolism, vol. 90, no. 3, pp. 1578-1582, 2005.

[28] J. B. Schwimmer, P. E. Pardee, J. E. Lavine, A. K. Blumkin, and S. Cook, "Cardiovascular risk factors and the metabolic syndrome in pediatric nonalcoholic fatty liver disease," Circulation, vol. 118, no. 3, pp. 277-283, 2008.

[29] C. Giraldez-Garcia, F. J. Sangros, A. Diaz-Redondo et al., "Cardiometabolic risk profiles in patients with impaired fasting glucose and/or hemoglobin Alc 5.7\% to 6.4\%: evidence for a gradient according to diagnostic criteria: the PREDAPS Study," Medicine (Baltimore), vol. 94, no. 44, Article ID e1935, 2015.

[30] D. J. Cuthbertson, M. O. Weickert, D. Lythgoe et al., "External validation of the fatty liver index and lipid accumulation product indices, using ${ }^{1} \mathrm{H}$-magnetic resonance spectroscopy, to identify hepatic steatosis in healthy controls and obese, insulinresistant individuals," European Journal of Endocrinology, vol. 171, no. 5, pp. 561-569, 2014.

[31] L. Fedchuk, F. Nascimbeni, R. Pais, F. Charlotte, C. Housset, and V. Ratziu, "Performance and limitations of steatosis biomarkers in patients with nonalcoholic fatty liver disease," Alimentary Pharmacology and Therapeutics, vol. 40, no. 10, pp. 1209-1222, 2014.

[32] M. A. Borman, F. Ladak, P. Crotty et al., "The Fatty Liver Index has limited utility for the detection and quantification of hepatic steatosis in obese patients," Hepatology International, vol. 7, no. 2, pp. 592-599, 2013.

[33] E. Vanni and E. Bugianesi, "Editorial: utility and pitfalls of Fatty Liver Index in epidemiologic studies for the diagnosis of NAFLD," Alimentary Pharmacology and Therapeutics, vol. 41, no. 4, pp. 406-407, 2015.
[34] M. Kozakova, C. Palombo, M. P. Eng et al., "Fatty liver index, gamma-glutamyltransferase, and early carotid plaques," Hepatology, vol. 55, no. 5, pp. 1406-1415, 2012.

[35] C.-L. Cheung, K. S. L. Lam, I. C. K. Wong, and B. M. Y. Cheung, "Non-invasive score identifies ultrasonography-diagnosed non-alcoholic fatty liver disease and predicts mortality in the USA," BMC medicine, vol. 12, p. 154, 2014.

[36] E. Lerchbaum, S. Pilz, T. B. Grammer et al., "The fatty liver index is associated with increased mortality in subjects referred to coronary angiography," Nutrition, Metabolism and Cardiovascular Diseases, vol. 23, no. 12, pp. 1231-1238, 2013.

[37] G. Calori, G. Lattuada, F. Ragogna et al., "Fatty liver index and mortality: the cremona study in the 15th year of follow-up," Hepatology, vol. 54, no. 1, pp. 145-152, 2011.

[38] I. Wakabayashi and T. Daimon, "A strong association between lipid accumulation product and diabetes mellitus in Japanese women and men," Journal of Atherosclerosis and Thrombosis, vol. 21, no. 3, pp. 282-288, 2014.

[39] C. Xia, R. Li, S. Zhang et al., "Lipid accumulation product is a powerful index for recognizing insulin resistance in nondiabetic individuals," European Journal of Clinical Nutrition, vol. 66, no. 9, pp. 1035-1038, 2012.

[40] D. Brisson, P. Perron, H. S. Kahn, D. Gaudet, and L. Bouchard, "The lipid accumulation product for the early prediction of gestational insulin resistance and glucose dysregulation," Journal of Women's Health, vol. 22, no. 4, pp. 362-367, 2013.

[41] J.-Y. Oh, Y.-A. Sung, and H. J. Lee, “The lipid accumulation product as a useful index for identifying abnormal glucose regulation in young Korean women," Diabetic Medicine, vol. 30, no. 4, pp. 436-442, 2013.

[42] H. S. Kahn, "The lipid accumulation product is better than BMI for identifying diabetes: a population-based comparison," Diabetes Care, vol. 29, no. 1, pp. 151-153, 2006.

[43] M. Bozorgmanesh, F. Hadaegh, and F. Azizi, "Diabetes prediction, Lipid accumulation product, and adiposity measures; 6-year follow-up: Tehran Lipid and Glucose Study," Lipids in Health and Disease, vol. 9, article no. 45, 2010.

[44] C. Zhong, W. Xia, X. Zhong et al., "Lipid accumulation product and hypertension related to stroke: a 9.2-year prospective study among Mongolians in China," Journal of Atherosclerosis and Thrombosis, vol. 23, no. 7, pp. 830-838, 2016.

[45] J. H. Kim, S. Y. Kwon, S. W. Lee, and C. H. Lee, "Validation of fatty liver index and lipid accumulation product for predicting fatty liver in Korean population," Liver International, vol. 31, no. 10, pp. 1600-1601, 2011.

[46] M. L. Tellechea, F. Aranguren, M. T. Martínez-Larrad, M. Serrano-Ríos, M. J. Taverna, and G. D. Frechtel, "Ability of lipid accumulation product to identify metabolic syndrome in healthy men from Buenos Aires," Diabetes Care, vol. 32, article no. e85, 2009.

[47] J.-K. Chiang and M. Koo, "Lipid accumulation product: a simple and accurate index for predicting metabolic syndrome in Taiwanese people aged 50 and over," BMC Cardiovascular Disorders, vol. 12, article 78, 2012.

[48] N. Motamed, S. Razmjou, G. Hemmasi, M. Maadi, and F. Zamani, "Lipid accumulation product and metabolic syndrome: a population-based study in northern Iran, Amol," Journal of Endocrinological Investigation, vol. 39, no. 4, pp. 375382, 2016.

[49] M.-C. Huang and C.-C. Chen, "Alcohol dependence in Taiwan: from epidemiology to biomedicine," Journal of Experimental \& Clinical Medicine, vol. 4, no. 2, pp. 108-112, 2012. 
[50] A. E. A. Joseph, S. H. Saverymuttu, S. Al-Sam, M. G. Cook, and J. D. Maxwell, "Comparison of liver histology with ultrasonography in assessing diffuse parenchymal liver disease," Clinical Radiology, vol. 43, no. 1, pp. 26-31, 1991.

[51] R. Haring, H. Wallaschofski, M. Nauck, M. Dörr, S. E. Baumeister, and H. Völzke, "Ultrasonographic hepatic steatosis increases prediction of mortality risk from elevated serum gamma-glutamyl transpeptidase levels," Hepatology, vol. 50, no. 5, pp. 1403-1411, 2009. 


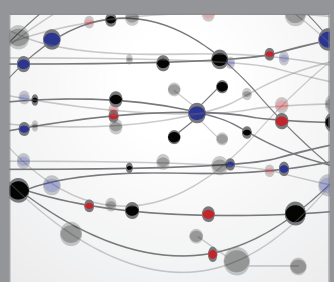

The Scientific World Journal
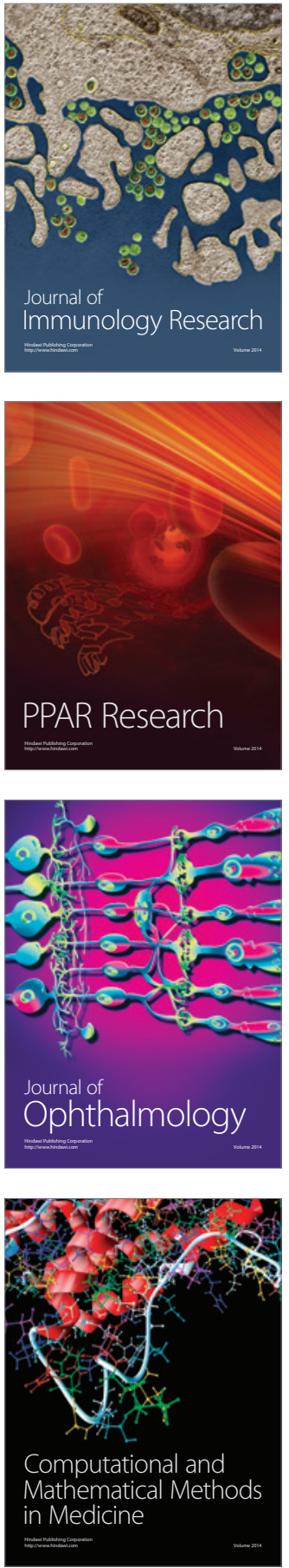

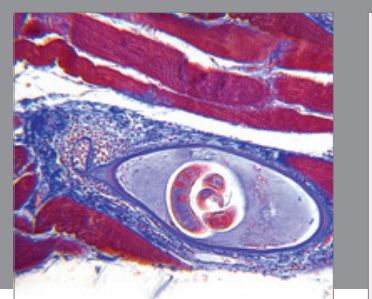

Gastroenterology Research and Practice
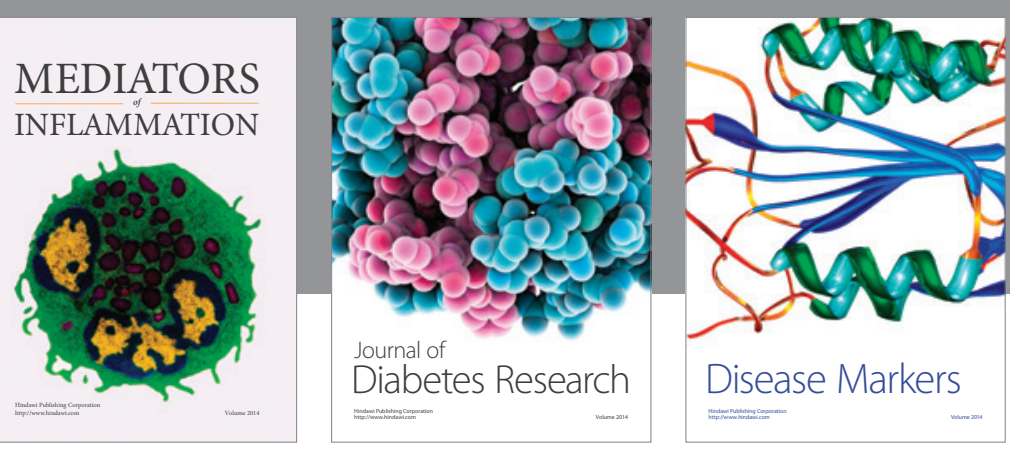

Disease Markers

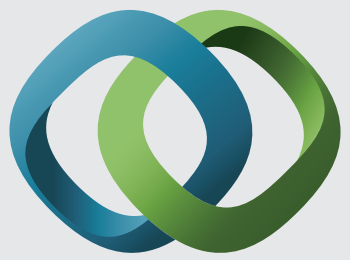

\section{Hindawi}

Submit your manuscripts at

https://www.hindawi.com
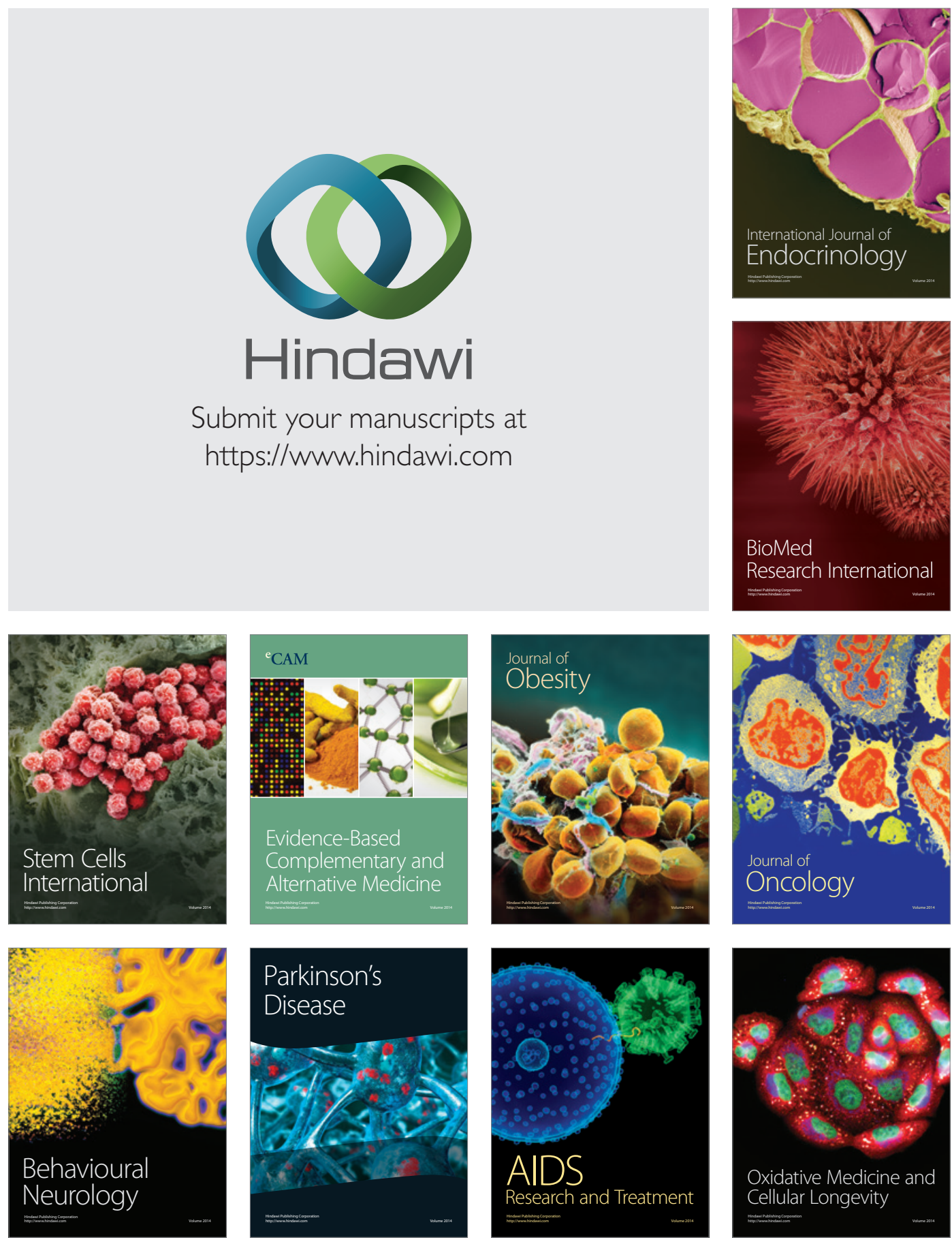